\title{
DEATH OF DR SAROLEA
}

\section{Olle Essvik}

https://books.google.com

I end up there by accident. I did a search on the Internet. For books on books.

The first thing that greets me is a search box. The text I type is processed through millions of published books. Older books that have been digitized. Books that have been scanned and added to a database in order to be searchable.

\section{Scanning}

The page of the book is turned towards the camera. It is scanned. It happens fast. Another page is scanned. Paper and ink become zeroes and ones. The machine turns the page. A new page. When all the pages have been scanned the computer collects them in a file. Digital formats. A dot and a letter combination. .pdf .txt .xml. 


\section{Google}

This is a digital copy of a book that was preserved for generations on library shelves before it was carefully scanned by Google as part of a project to make the world's books discoverable online.

It has survived long enough for the copyright to expire and the book to enter the public domain. A public domain book is one that was never subject to copyright or whose legal copyright term has expired.

Whether a book is in the public domain may vary country to country. Public domain books are our gateways to the past, representing a wealth of history, culture and knowledge that's often difficult to discover.

Marks, notations and other maiginalia present in the original volume will appear in this file - a reminder of this book's long journey from the publisher to a library and finally to you.

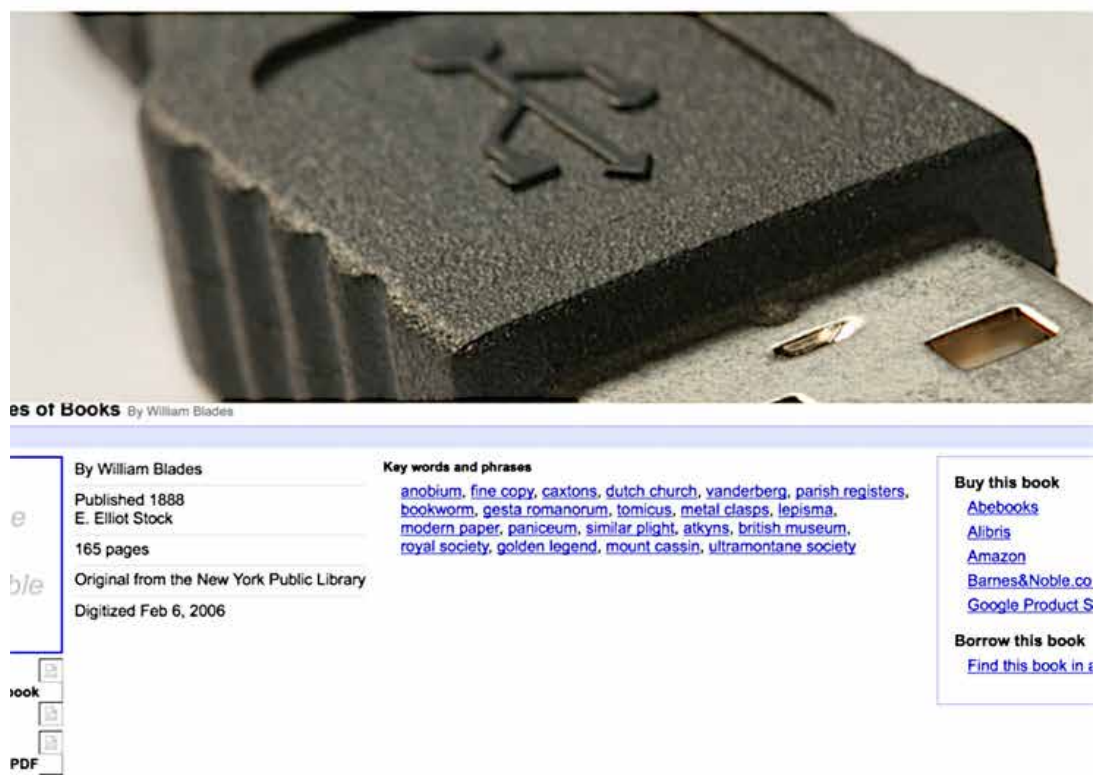

Figure 1 
Enemies of books. Written by William Blades, published in 1881 . A book on the decay of books. The enemies of physical books - fire, water, gas, the bookworm, bigotry, etc (figure 1). I print out the book. White pages and black marks. No identity. The software scans stains and scratches as letters. Physical traces are digitized.

I find the original on the Internet. A second hand bookshop in London has a copy. I order the book. An impersonal form and payment directions. Credit card. The book is expensive, but in unusually good condition. A few weeks later I receive a package smelling of paper and incense (figure 2).

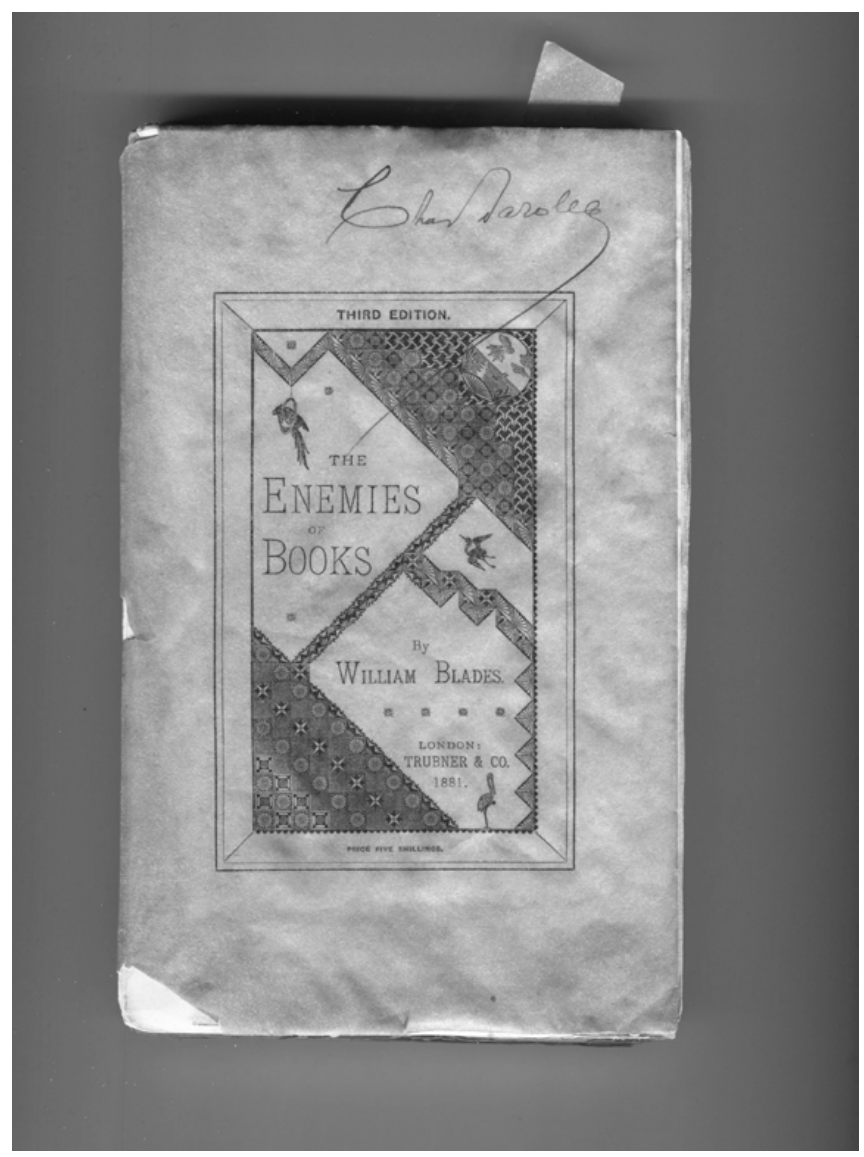

Figure 2 
The book is yellowed and the edges are dark brown with dirt. The paper has been bleached by time and the sun. The cover is worn. A thin sheet of paper protects the book. Looks like greaseproof paper. A simple binding.

The third edition. The spine is frayed, the pages are loose and the thread holding them together is visible. Each page has unique stains and creases. The edges are uneven, uncut.

A signature on the cover page. The owner of the book. Inside the book are some newspaper clippings, an obituary of Dr. Sarolea (figure 3). The book belonged to him. Another clipping tells the story of his book collection, consisting of 300000 volumes, weighing 100 ton. It is described as one of the wonders of the world. Dr. Sarolea is dead but his book collection is alive. A note about the good condition of the book on the cover page. Added by the bookshop. A rubbed out price. Another signature. Unknown. The book ends with date, probably jotted down by Dr. Sarolea. Possibly the date when he finished the book.

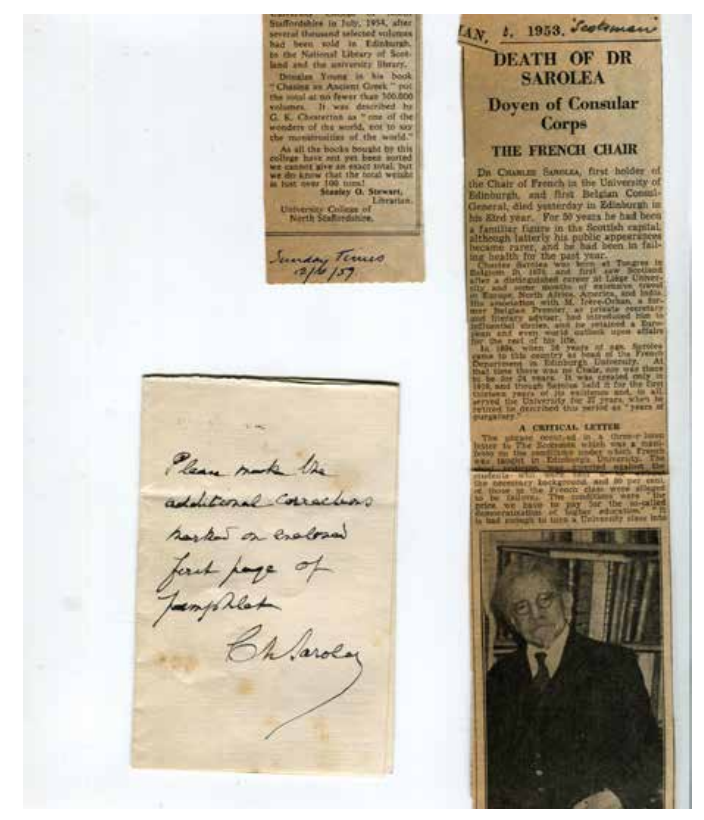

Figure 3 
I read the book. It is still not mine. The stains and traces are foreign. I can feel the presence of the former owners. People I do not know. Every time I pick it up it becomes more mine. The smell of my apartment is mixed with the incense and my invisible fingerprints are now added to the older marks.

I read the digitized book. The digital file begins with information on how the book has been scanned by Google. The date of the scanning and information on Google books (figure 4).

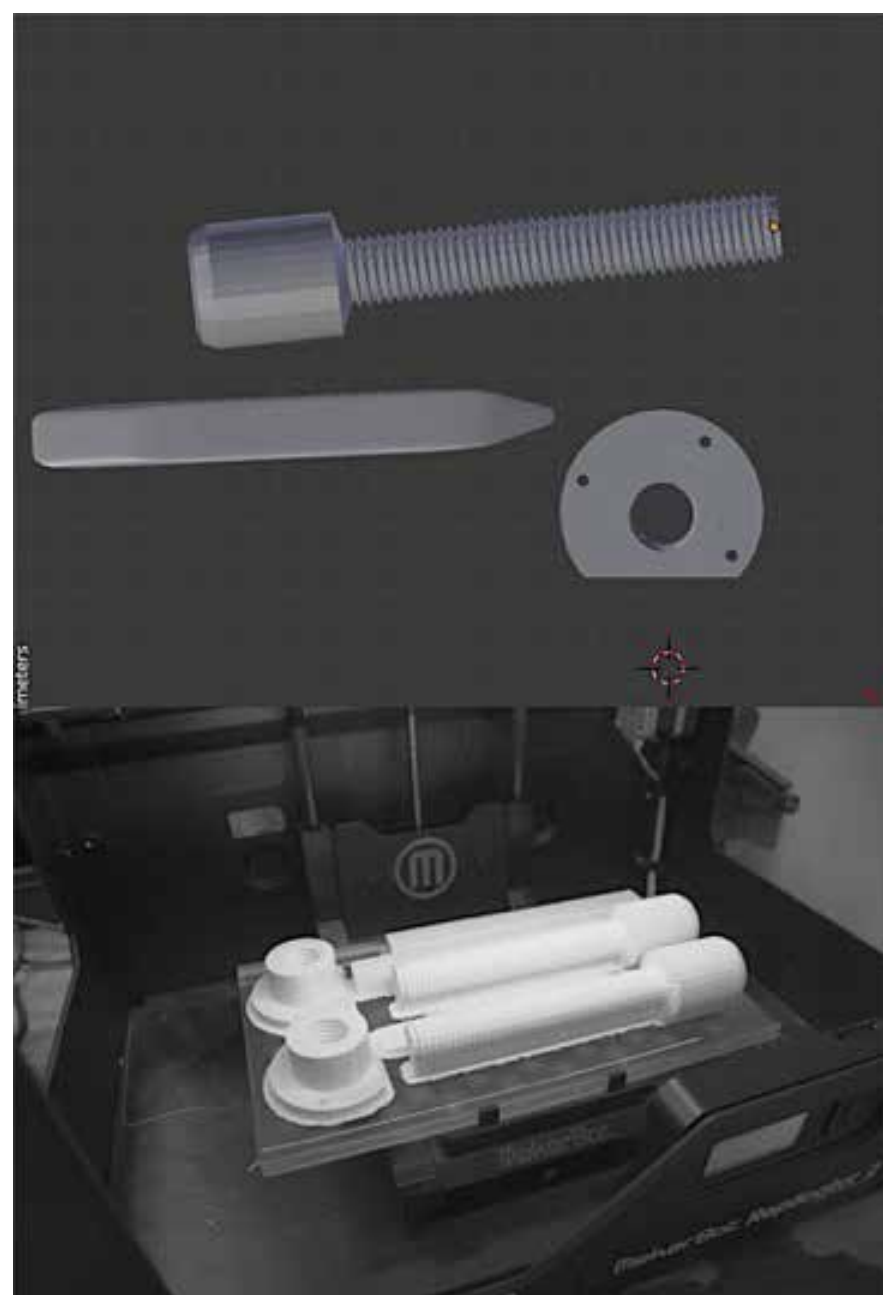

Figure 4 
I recreate the book. I let a $3 \mathrm{D}$-printer print out the tool. I want to test if it works. It does (figure 5).

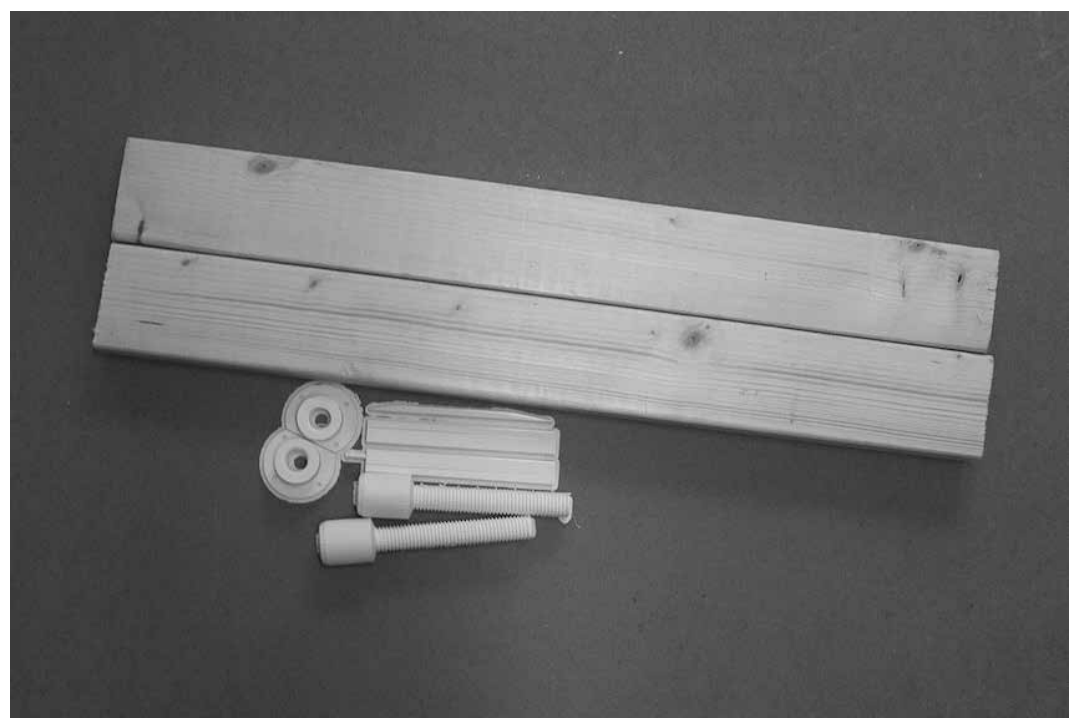

Figure 5

At night while I sleep the file is processed by the printer, typing out layers of plastic. The digital file is based on tools that have been used for hundreds of years. Combining and modifying the old techniques I create the necessary tools. The components are mounted on a piece of board (figure $6,7,8$ ). I print out and bind the book (figure 9, 10, 11, 12). I upload the files to the Internet. 

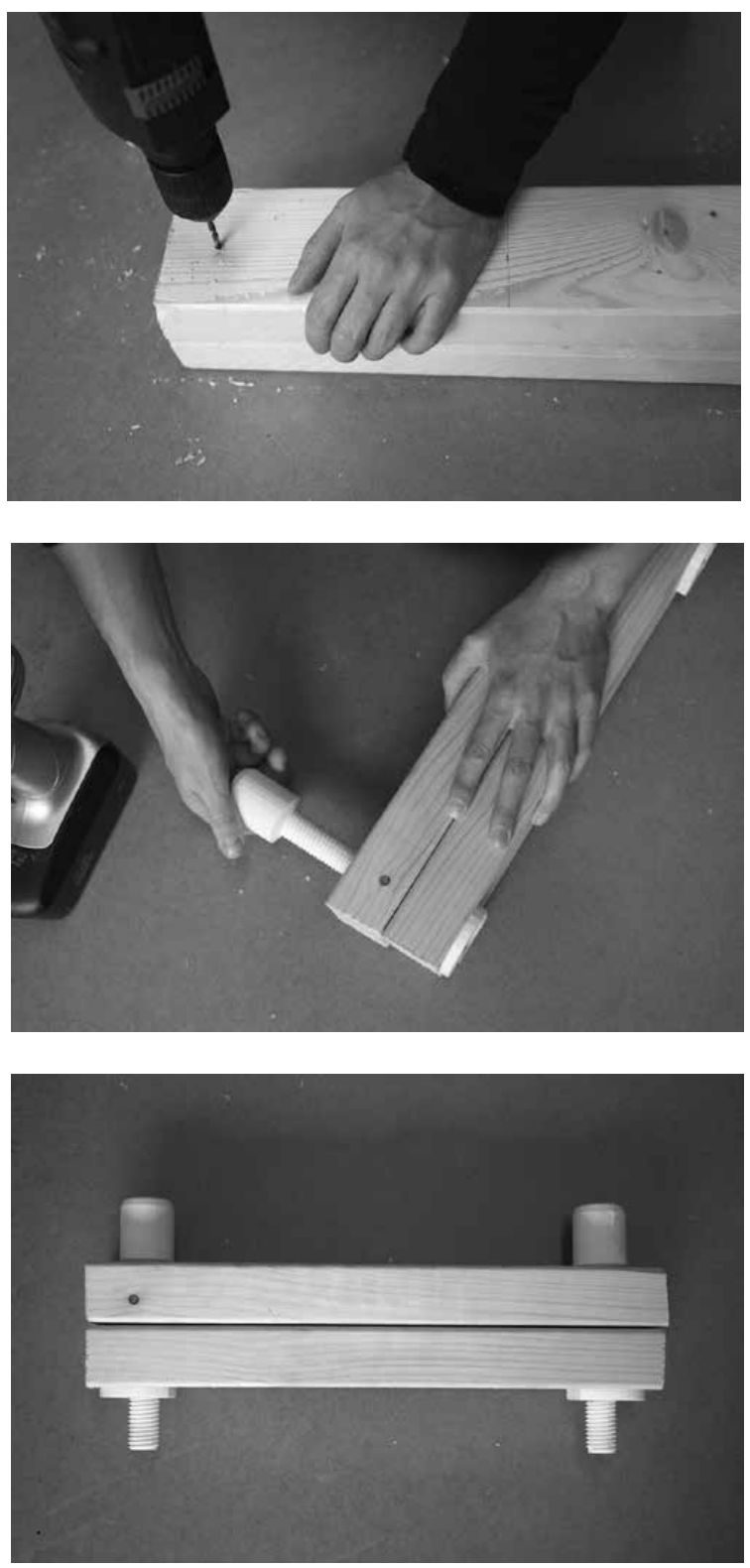

Figure 6 


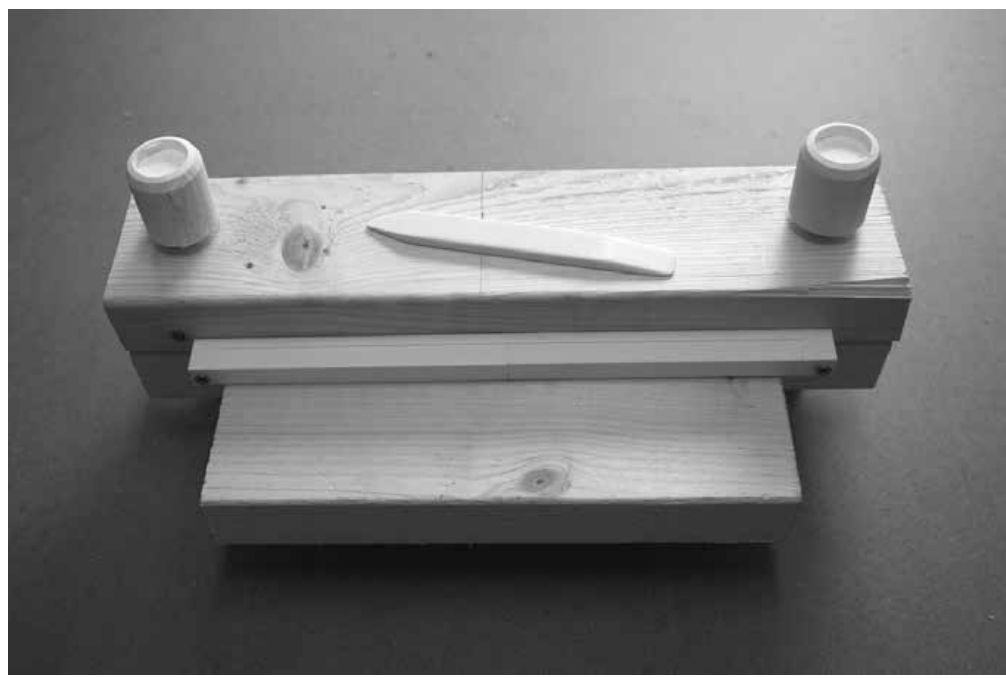

Figure 7

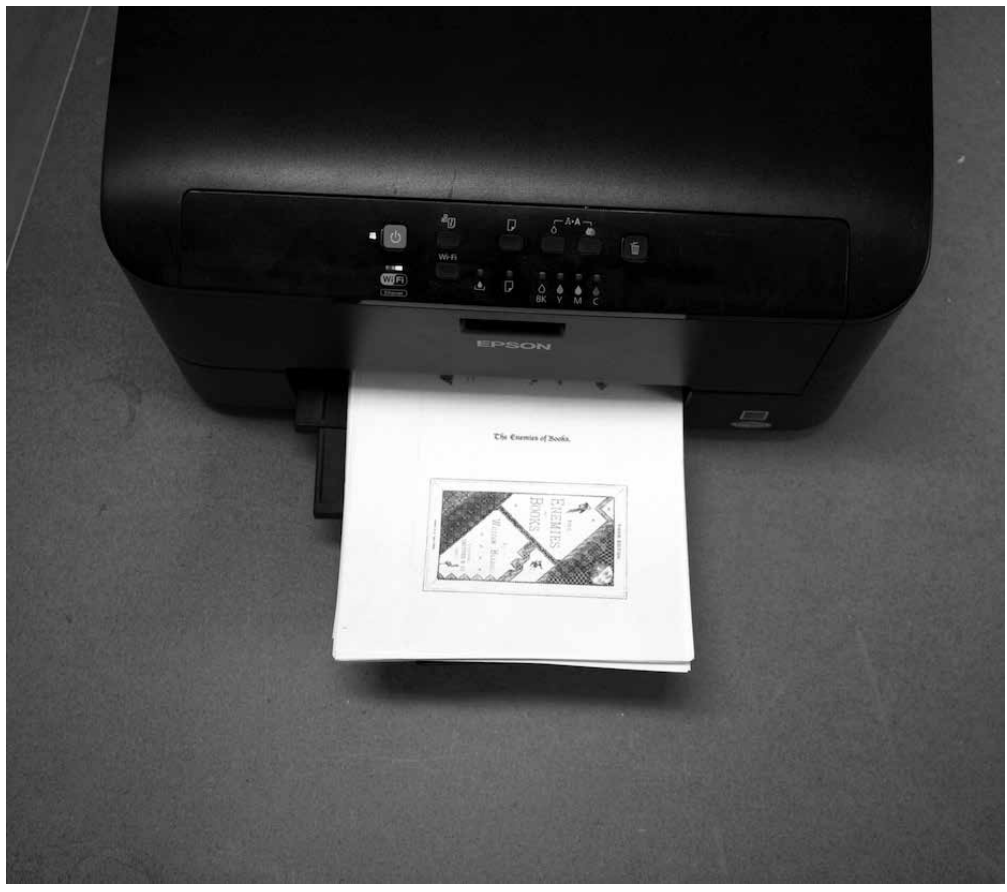

Figure 8 

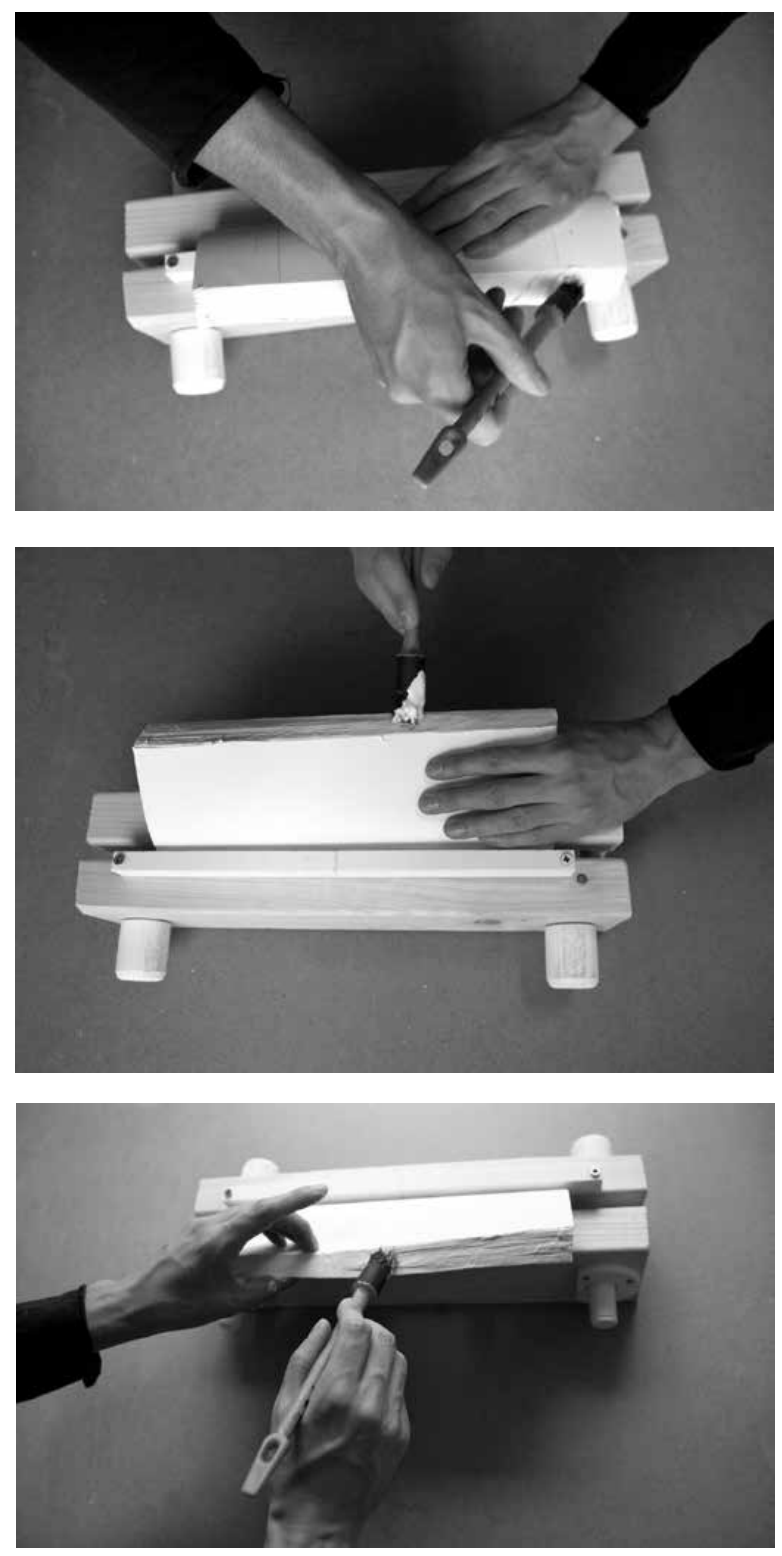

Figure 9 

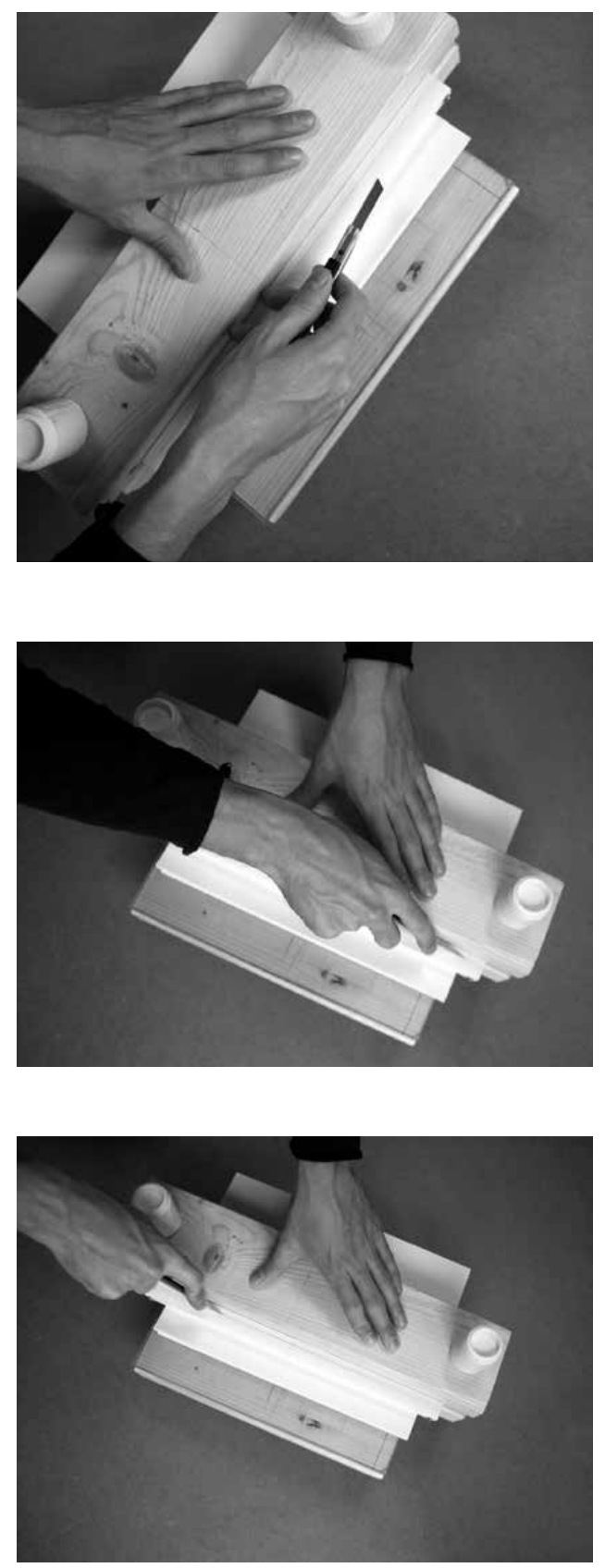

Figure 10 

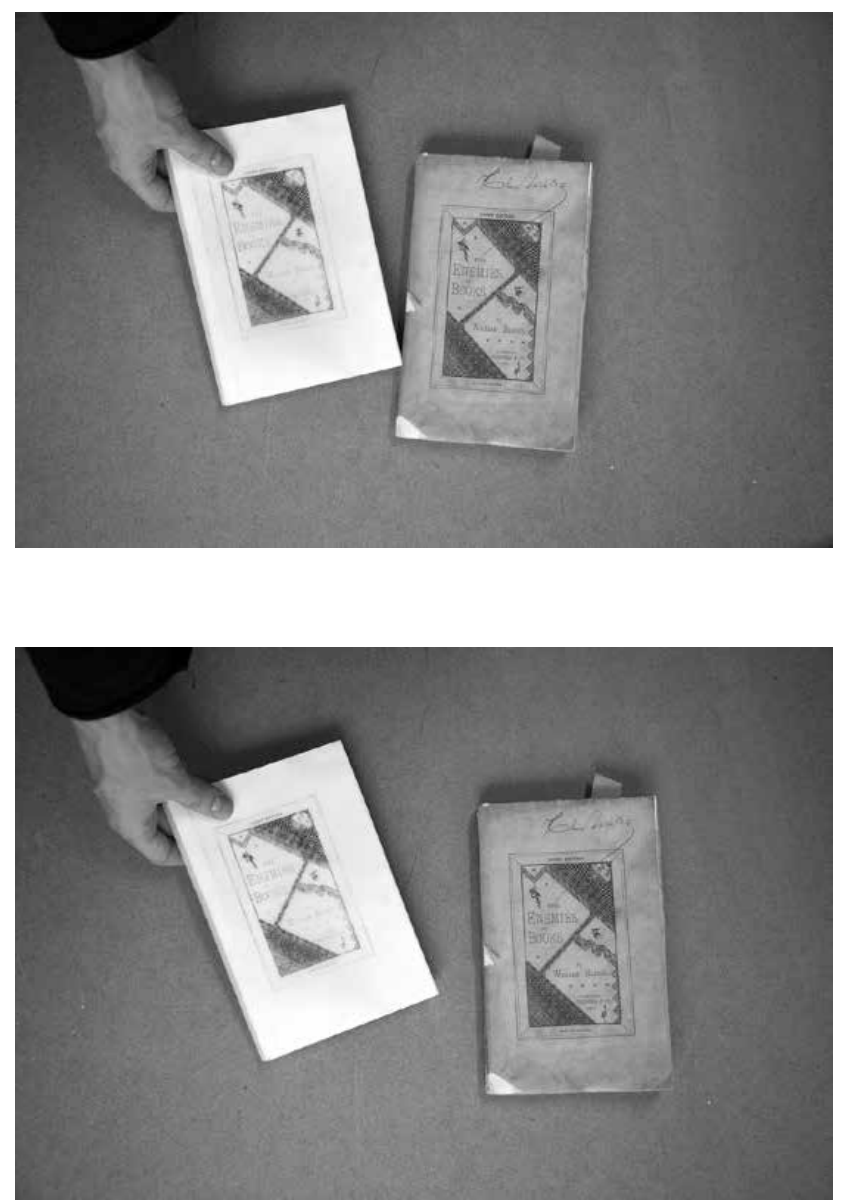

Figure 11 